\title{
Towards Immersive Software Engineering Education
}

\author{
Filipe Fernandes \\ Computer Science Department \\ IF Sudeste MG - Federal Institute Southeast of Minas Gerais \\ Manhuaçu, MG - Brazil \\ filipe.arantes@ifsudestemg.edu.br, ffernandes@cos.ufrj.br
}

\author{
Cláudia Werner \\ System Engineering and Computer Science Department \\ COPPE/UFRJ - Federal University of Rio de Janeiro \\ Rio de Janeiro, RJ - Brazil \\ werner@cos.ufrj.br
}

\begin{abstract}
This paper proposes to present an approach to obtain evidence about the influence of immersion in improving learning outcomes in Software Engineering. An immersive platform has been developed and a preliminary study with 6 subjects has been conducted to identify usability issues in an immersive application prototype to support the comprehension of Object-Oriented Paradigm theoretical concepts. Although the main problems are related to the affordances of the virtual world and the interaction between user and application, there was evidence that immersive technologies have the potential to support Software Engineering Education.
\end{abstract}

Keywords - immersive learning, immersive technologies, learning outcomes, software engineering education

\section{INTRODUCTION}

Immersive Learning (iLearning) is a recent area of research aimed at contributing to the teaching and learning process through immersive technologies such as Augmented and Virtual Reality (AVR). It goes beyond just using immersive technologies in education. iLearning enhances the mental state, known as sense of presence, as one of the main factors that influence the improvement of learning outcomes. In the technical literature, iLearning is used as a method to support the teaching and learning process in various areas of knowledge. However, Software Engineering Education (SEE) lacks studies to obtain evidence on improving learning outcomes through immersive technologies.

\section{Motivation}

The main question that motivates this research is how to use immersive technologies to improve learning outcomes in Software Engineering. The great challenge of this research is to create mechanisms through immersive technologies, among them to enable to overcome the main obstacle of SEE: the teaching of practice.

Although theoretical approaches are well applied in universities, SEE needs more practical, dynamic and engaging teaching approaches than just lectures. It needs the active participation of students in real projects, that is, they need to live experiences, which are provided only by the professional environment of industry [1]. Therefore, SEE should engage students in the experience of professional practice, so that they can understand which practices and techniques are useful in different situations. However, due to the time and scope constraints inherent in the academic environment, there are few opportunities to explore the many facets of Software Engineering (SE). As a result, universities inherit the challenge of preparing software engineers with the skills and competencies required from industry [2].

\section{RELATED WORK}

A virtual world proposed by [3] was used to enhance software engineering education by exploiting communication and collaboration tools to teach practices such as problem solving, plan formulation, interpretative analysis, and adaptation to rapid change, in a virtual office provided by Second Life (SL). In addition, in order to evaluate the effects of SL when playing games, the authors developed the SL version of two games to introduce software engineering practices: Groupthink and SimSE. The former focuses on teaching software specification practices, and the latter aims to train project management skills. Likewise [3], [4] used a 3D virtual world based on Open Wonderland to support a workshop activity based on agile software development processes. This workshop enables students to take part in this activity despite their geographical distribution. [5] developed the educational game in Second Life, based on the SimSE game developed at University of California at Irvine. It provides the students the simulated experience as software engineers, especially the communication and collaboration with other team members in a project.

It is noted that experiences through immersive technologies are not explored, as well as other aspects of SE.

\section{PROPOSED SOLUTION}

Considering the challenges of teaching and the lack of empirical evidence of iLearning applied to SE, this research has been moving towards an Immersive Software Engineering Education approach, named iSEE. The aim of the approach is to provide immersive experiences as a mechanism for improving learning outcomes in various fields of SE through an immersive teaching platform. Fig. 1 presents an overview of the approach.

The user accesses the virtual world and selects some application ( $a p p)$ according to the instructional purpose. The application is then downloaded and installed on the user's device. Considering the VR ecosystem, applications are developed for Virtual Reality Platforms and some platforms limit the use of immersive devices. Therefore, the platform will only provide applications compatible with the VR platform on which it is being accessed by the user. Applications are published by developers through Developers and Users Platform. This web platform allows the compiled application file to be registered and persisted. Through webservice, this platform makes available the platform applications to the virtual world.

A prototype of the immersive platform was developed, as well as an immersive application aiming to support the understanding of theoretical concepts of the Object-Oriented Paradigm (OOP). This application, named OO Game VR, represents one of several applications that can be made available on the platform and foster immersive learning in the various fields of SE.

The idea of this game is to build three-dimensional drawings from primitive cube and sphere visuals based on the concepts of OOP. This game is designed to be compatible with Oculus Rift. 


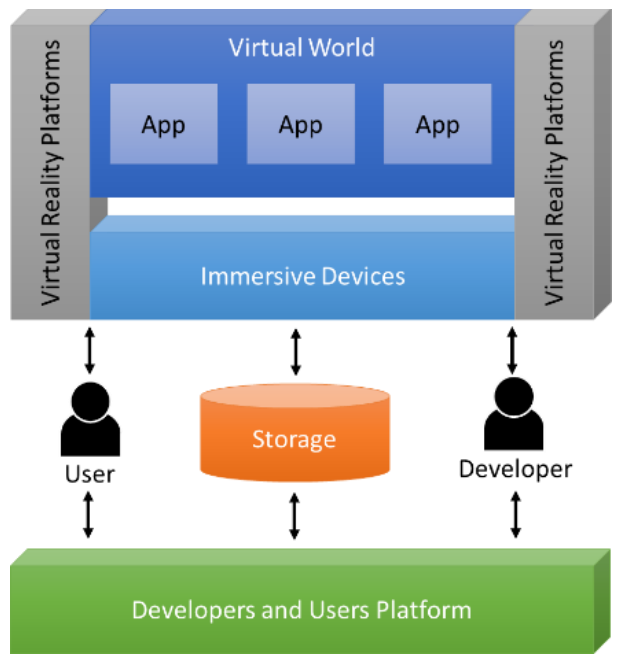

Fig. 1. Immersive Software Engineering Education Approach Overview.

\section{Preliminary Results}

Considering the scope of a doctoral research, it would be impracticable to conduct a controlled experiment that can assess all the knowledge required by a software engineer. Therefore, a preliminary study was conducted to identify $\mathrm{OO}$ Game VR usability problems and correct them prior to observing the influence of immersion on learning. According to the objective of the evaluation, the heuristics for Virtual Reality applications proposed by [6] were adopted as metrics.

In the preliminary study, 6 students from a Federal Institute of Education, Science and Technology participated in the heuristic evaluation. Firstly, training on usability evaluation of virtual environments was conducted, as well as the use of the heuristic evaluation method of Virtual Reality (VR) applications [6]. Then, the OO Game VR assessment was conducted individually with the students. After completing the profile questionnaire, each student freely used the immersive application, performed 11 tasks and then evaluated the application according to the twelve heuristics proposed by [6].

In order to homogenize the result of the degrees of severity by heuristic and support in identifying the most critical, a weight was assigned to each degree; varying from the least critical degree with 1 to the most critical value 4 . As result, the heuristics $\mathrm{H} 10, \mathrm{H} 07, \mathrm{H} 03$ and $\mathrm{H} 08$ had the most critical degrees of severity (see Fig. 2).

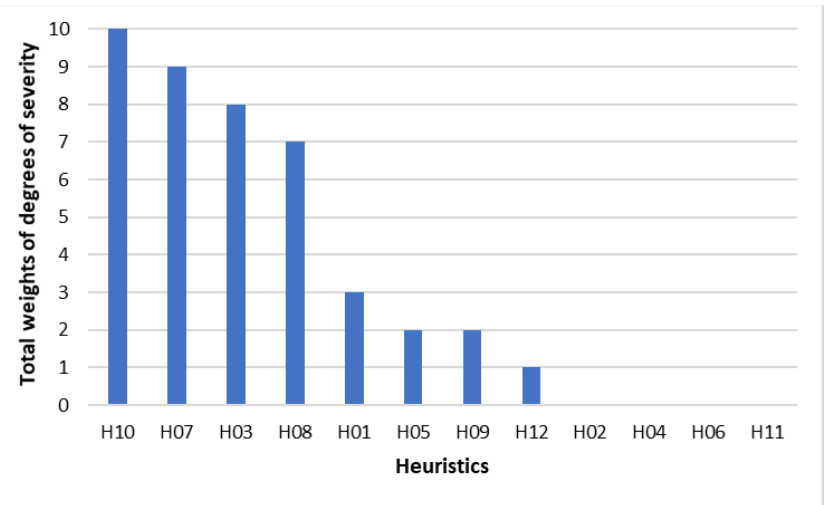

Fig. 2. Weight of degrees of severity per heuristic.
The heuristics indicated as problematic are related to the characteristic that the game has in assisting the user in manipulating the elements of the virtual world, as well as in the interaction between user and application through immersive devices. Despite these problems, all subjects were able to complete the 11 tasks. It can also be observed that the heuristics related to engagement ( $\mathrm{H} 01)$ and sense of presence (H12) presented low degrees of severity. Although not being the focus of this study, this result shows that the most critical heuristics in this assessment have little influence on engagement and sense of presence.

\section{CONCLUSION}

This paper aims to present an approach to Software Engineering Education based on Immersive Learning, named Immersive Software Engineering Education (iSEE). It aims to obtain empirical evidence about the influence of immersion on improving learning outcomes. Considering that Software Engineering involves a range of technical and non-technical knowledge and skills required from engineers, an immersive platform was developed to encompass a variety of immersive applications with different instructional purposes, as well as a combination of immersive devices, each with specific characteristics that influence users' sense of presence.

A preliminary study was conducted to identify usability issues in an immersive application prototype that supports the comprehension of the Object-Oriented Paradigm. The results show that the main usability issues are related to affordances of the virtual world, as well as the interaction between user and application. As future work, these problems will be addressed, other applications will be developed, and a controlled study will be conducted to obtain evidence on the influence of immersion on improving Software Engineering learning outcomes.

\section{ACKNOWLEDGMENT}

This research was partly supported by CAPES, CNPq and IF Sudeste MG. We wish to thank the students who participated in the training and heuristic evaluation.

\section{REFERENCES}

[1] M. Moore and C. Potts, "Learning by doing: Goals and experiences of two software engineering project courses," in Conference on Software Engineering Education, 1994, pp. 151-164.

[2] M. R. Marques, A. Quispe, and S. F. Ochoa, "A systematic mapping study on practical approaches to teaching software engineering," in 2014 IEEE Frontiers in Education Conference (FIE) Proceedings, 2014, pp. 1-8.

[3] E. Ye, C. Liu, and J. A. Polack-Wahl, "Enhancing software engineering education using teaching aids in 3-D online virtual worlds," in Frontiers in education conference-global engineering: knowledge without borders, opportunities without passports, 2007. FIE'07. 37th annual, 2007, pp. T1E-8.

[4] D. Parsons and R. Stockdale, "Cloud as context: Virtual world learning with open wonderland," in Proceedings of the 9th World Conference on Mobile and Contextual Learning, Malta, 2010, pp. 123-130.

[5] T. Wang and Q. Zhu, "A software engineering education game in a 3$\mathrm{D}$ online virtual environment," in Education Technology and Computer Science, 2009. ETCS'09. First International Workshop on, 2009, vol. 2, pp. 708-710.

[6] A. Sutcliffe and B. Gault, "Heuristic evaluation of virtual reality applications," Interact. Comput., vol. 16, no. 4, pp. 831-849, Aug. 2004 